\title{
Management of systolic anterior motion after mitral valve repair: An algorithm
}

\author{
Robin Varghese, MD, MS, FRCSC, Anelechi C. Anyanwu, MD, MSc, FRCS, Shinobu Itagaki, MD, \\ Federico Milla, MD, Javier Castillo, MD, and David H. Adams, MD
}

Objective: To evaluate the effectiveness and outcomes of an intraoperative and postoperative algorithm for managing systolic anterior motion (SAM) after mitral valve repair (MVRr).

\begin{abstract}
Methods: All consecutive patients who underwent MVRr for degenerative disease from January 2002 to June 2011 were included, with the data collected retrospectively. Patients who underwent MVRr for primary SAM were excluded from the study. Patients who developed SAM after the repair were systematically treated according to the algorithm. The intraoperative algorithm first involved medical management techniques, followed by surgical correction for significant SAM (mild or greater mitral regurgitation, left ventricular outflow tract gradient $>50 \mathrm{~mm} \mathrm{Hg}$ ). The postoperative algorithm focused on medical management and symptoms to guide the treatment decisions.
\end{abstract}

Results: The overall in-hospital incidence of SAM was 6.6\% (52/785). In 41 patients, SAM was identified in the operating room, and in 11 patients, it was found postoperatively on the predischarge echocardiogram. Of the 41 patients with intraoperative SAM, $35(85.4 \%)$ had resolution with medical management and $6(14.6 \%)$ required surgical repeat repair while in the operating room. No patient required mitral valve replacement for persistent SAM. Postoperatively, 11 new cases were identified, and 7 cases of resolved intraoperative SAM recurred. These postoperative cases of SAM were managed according to the postoperative SAM algorithm. At last follow-up, 17 (94.4\%) of 18 patients had resolution of SAM and $1(5.6 \%)$ patient had mild SAM (less than mild mitral regurgitation, peak left ventricular outflow tract gradient $<50 \mathrm{~mm} \mathrm{Hg}$ ) and were asymptomatic. No patients with postoperative SAM required reoperation after their initial surgery. The median echocardiographic follow-up was 1.3 years. During follow-up, 1 early death (noncardiac) and 2 late deaths (1 noncardiac, 1 of unknown etiology) occurred.

Conclusions: SAM is a relatively frequent complication after MVRr and can occur intraoperatively or postoperatively. A systematic approach addressing perioperative SAM after MVRr yields excellent mid-term results. (J Thorac Cardiovasc Surg 2012;143:S2-7)

In the current era of mitral valve repair (MVRr), systolic anterior motion (SAM) of any severity occurs in $4 \%$ to $10 \%$ of cases. ${ }^{1,2}$ The degree of SAM after MVRr can vary from mild to severe and is typically associated with mitral regurgitation (MR) with left ventricular outflow tract (LVOT) obstruction. Despite a number of techniques designed to avert SAM, it still occurs at a significant incidence, requiring surgeons to be cognizant of its possibility and approaches to managing

\footnotetext{
From the Department of Cardiothoracic Surgery, Mount Sinai Medical Center, New York, NY.

Disclosures: Robin Varghese, Anelechi C. Anyanwu, Shinobu Itagaki, Federico Milla, Javier Castillo, and David H. Adams have nothing to disclose with regard to commercial support.

Presented at The American Association for Thoracic Surgery Mitral Conclave, New York, New York, May 5-6, 2011.

Received for publication June 4, 2011; revisions received Jan 2, 2012; accepted for publication Jan 23, 2012

Address for reprints: Robin Varghese, MD, MS, FRCSC, Department of Cardiothoracic Surgery, Mount Sinai Medical Center, 1190 Fifth Avenue, Box 1028, New York, NY 10029 (E-mail: robin.varghese@mountsinai.org).

$0022-5223 / \$ 36.00$

Copyright (c) 2012 by The American Association for Thoracic Surgery doi:10.1016/j.jtcvs.2012.01.063
}

it. Although most cases of SAM resolve with medical management, situations exists in which a patient requires early or delayed reoperation for SAM, making this an issue that mitral valve surgeons should be comfortable treating. ${ }^{2}$ Aside from the basic medical approach to the management of SAM, knowing when to intervene perioperatively has not been clear. We believe that with an appropriate algorithm for the perioperative management of SAM, one can be better equipped to systematically address the problem and know when and how to intervene. We provide a review of SAM, followed by our algorithm for the management of SAM and our results using this algorithm.

\section{SAM: WHAT IS IT?}

SAM is defined as displacement of the distal portion of the anterior leaflet of the mitral valve toward the LVOT during systole. ${ }^{3}$ The primary lesion resulting in SAM after MVRr is a mismatch between the mitral valve annular dimension and the amount of leaflet tissue present. A number of mechanisms have been described to account for SAM 


\section{Abbreviations and Acronyms \\ LVOT $=$ left ventricular outflow tract \\ $\mathrm{MR}=$ mitral regurgitation \\ $\mathrm{MVRr}=$ mitral valve repair \\ $\mathrm{SAM}=$ systolic anterior motion}

with the 2 most predominant mechanisms being the "venturi effect" and the "drag effect." Both mechanisms describe the anterior leaflet being drawn into the outflow tract either by a pulling (venturi) or pushing (drag) phenomenon. ${ }^{4}$ As the left ventricle contracts and ejects blood through the outflow tract and into the aorta, it creates drag (push) on redundant anterior leaflet tissue, drawing the tip of the anterior leaflet into the outflow, creating turbulence to flow that further creates a venturi (pulling) effect on the anterior leaflet and the potential for MR. This mechanism for SAM typically occurs secondary to MVRr for degenerative disease. SAM can also occur primarily in cases of hypertrophic cardiomyopathy, in which a thickened left ventricular septum in addition to abnormal chordal attachments to the anterior leaflet in the presence of a hypertrophied and hyperdynamic ventricle can result in SAM. In this case, the drag phenomenon is believed to play a larger role than the venturi effect. In the present report, we have focused on SAM that occurs secondary to MVRr surgery for degenerative disease.

A number of risk factors exist that predispose a patient to SAM. These can be divided into patient factors and technical factors related to the repair. Patient risk factors include a narrow aortomitral angle, a bulging left ventricular septum, and a hyperdynamic small left ventricle. ${ }^{5}$ Technical repair factors include excessive posterior leaflet tissue $(>15$ $\mathrm{mm}$ ) and an annuloplasty ring that is undersized relative to the size of the anterior leaflet. ${ }^{6}$

\section{METHODS}

From January 2002 to June 2011, 785 consecutive patients who underwent MVRr for degenerative disease were included. Data were collected in a retrospective manner. Patients with primary systolic anterior motion as a result of hypertrophic obstructive cardiomyopathy or previous mitral valve surgery were excluded. Patients who underwent concomitant tricuspid valve repair or concomitant coronary artery bypass surgery were included. Intraoperatively and postoperatively, the patients who developed SAM were treated according to a prespecified SAM management algorithm (Figures 1 and 2). Intraoperatively, SAM was diagnosed by cardiac anesthesiologists certified in echocardiography and was defined as any portion of the anterior leaflet prolapsing into the LVOT. The degree of SAM was classified into 2 categories: mild SAM (LVOT gradient $<50$ $\mathrm{mm} \mathrm{Hg}$ and/or less than mild MR) and significant SAM (LVOT gradient $>50 \mathrm{~mm} \mathrm{Hg}$ and/or mild MR or greater). A number of repair techniques were used to repair the mitral valve, including leaflet resection, leaflet sliding plasty, and placement of neochordae. All patients had either a full or partial annuloplasty ring placed. All patients were followed up with serial echocardiography and clinical evaluation by our team and subsequently by the patient's cardiologist. Patients found to have significant SAM postoperatively were treated according to the algorithm and underwent serial echocardiograms until resolution of significant SAM was evident.

\section{Algorithm for SAM}

Managing SAM perioperatively requires a systematic approach to the problem (Figure 1). Intraoperatively, minimizing the risk of SAM begins with technically adequate repair. This includes ensuring that the posterior leaflet height above the coaptation zone is not excessively tall $(>15 \mathrm{~mm})$ and that the annuloplasty ring is appropriately sized. In cases of posterior leaflet resection, a sliding leaflet technique can be used to shorten the posterior leaflet height. ${ }^{7} \mathrm{We}$ also use the intraoperative "ink-test" to assess the coaptation depth of the anterior leaflet. ${ }^{8}$

If SAM is seen initially when weaning from bypass, our recommendation is to first use the medical interventions listed in the algorithm to optimize the physiology. If persistent SAM is present, the degree of SAM and the structural appearance of the valve on echocardiography is important. If the posterior or anterior leaflet tissue is clearly excessive, regardless of the response to medical interventions, we would have a low threshold for resuming bypass and surgical correction because the patient will likely remain with a substrate for future SAM. In young patients, especially those who had asymptomatic MR, we have a very low threshold for surgical correction of SAM, irrespective of the response to medical interventions. If mild SAM is present, defined as less than mild MR and a LVOT gradient of less than $50 \mathrm{~mm} \mathrm{Hg}$, we usually use no additional surgical intervention and proceed to reversal of heparin and decannulation. An echocardiogram should be done before hospital discharge to confirm the SAM does not recur or worsen when the patient is ambulatory. If the SAM is physiologically significant, defined as mild MR or greater and/or a LVOT gradient greater than $50 \mathrm{~mm} \mathrm{Hg}$, we recommend reinstituting cardiopulmonary bypass and performing surgical repair.

Managing SAM that is discovered after the patient has left the operating room, but before discharge, is more challenging and requires a more conservative approach (Figure 2). If mild SAM is present with less than mild MR and a LVOT gradient of less than $50 \mathrm{~mm} \mathrm{Hg}$, we would increase the dosage of oral $\beta$-blockers as tolerated and avoid the use of diuretics and antihypertensive agents for systolic blood pressure less than 135 $\mathrm{mm} \mathrm{Hg}$. If improved the patient would be discharged with a repeat echocardiogram at the patient's routine follow-up appointment in 6 weeks. If the SAM results in more than mild MR or a LVOT gradient greater than $50 \mathrm{~mm} \mathrm{Hg}$ exists, we would institute the above-stated medical treatments and repeat the echocardiogram in 48 hours. If resolved, again, the patient would be discharged home with a follow-up examination within 6 weeks.

If, however, the repeat transthoracic echocardiogram reveals persistent SAM with more than mild MR and/or a LVOT gradient greater than $50 \mathrm{~mm}$ $\mathrm{Hg}$, one should consider early reoperation according to the symptoms, severity of excess leaflet tissue prolapsing into the LVOT, and the patient's comorbidities. In these patients, we invariably discharge them home with medical therapy and perform a repeat transthoracic echocardiogram within 2 to 6 weeks. If the SAM is still significant, we would recommend elective reoperation if the patient is without major comorbidities.

\section{RESULTS}

The overall incidence of SAM after MVRr was $6.6 \%$ (52/785). The types of valve dysfunction are listed in Table 1. The repair techniques varied, and a lesionspecific approach was used to address the various pathologies. ${ }^{9}$

Of the 52 patients with SAM, 41 (78.8\%) were identified in the operating room, and 11 new (not identified intraoperatively) cases $(21.2 \%)$ were identified on the predischarge 


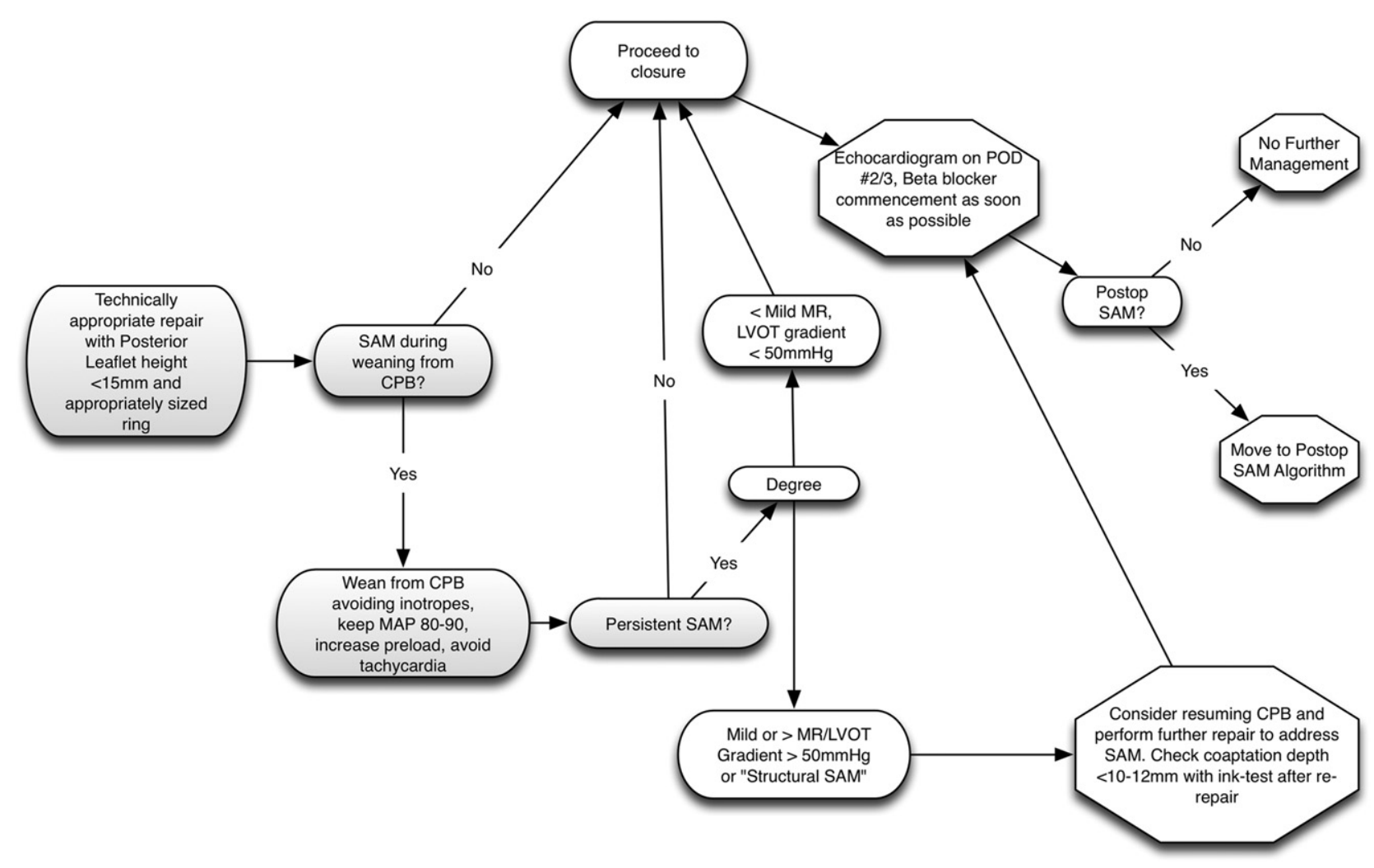

FIGURE 1. Management of intraoperative systolic anterior motion.

echocardiogram. Using intraoperative medical therapy (increased mean arterial pressure, slow heart rate, increased preload, and avoidance of inotropes) $35(85.3 \%)$ of 41 patients with intraoperative SAM had resolution or the SAM was downgraded to mild, hence not requiring additional intraoperative intervention. Despite intraoperative medical therapies, $6(14.7 \%)$ of the 41 patients had persistent significant SAM after discontinuation of cardiopulmonary bypass. In these 6 patients, surgical repeat repair of the valve was performed during the same operation. The details of these 6 patients' initial repair and the repeat repair techniques used to address SAM are listed in Table 2. In all cases of repeat repair, shortening of the posterior leaflet height was performed, in addition to other techniques. In those patients who required surgical repeat repair during the same operation for SAM-all cases of significant SAM resolved after repeat repair was performed. No patient required mitral valve replacement for persistent and significant SAM. The patients who developed SAM intraoperatively were identified, and their postoperative care was altered to ensure adequate medical management was instituted postoperatively to avoid worsening or recurrence of SAM. Higher mean arterial pressures $(75-90 \mathrm{~mm} \mathrm{Hg}$ ) were allowed, $\beta$-blocker therapy was instituted when possible, and aggressive diuresis was avoided during the patients' recovery.
Postoperatively, all patients underwent a predischarge echocardiogram between postoperative day 3 and the day of discharge. Of the 35 patients with intraoperative SAM, 7 showed recurrence on the predischarge echocardiogram. These patients were all asymptomatic at the time. The postoperative aspect of the SAM algorithm was then instituted for these patients. Of these 7 patients, 1 case involved a patient who had already undergone surgical intraoperative repeat repair for SAM (patient 6 listed in Table 1) during the initial operation. The LVOT gradient on the predischarge echocardiogram was measured at $52 \mathrm{~mm} \mathrm{Hg}$ with mild MR. After continued medical management and avoidance of diuretics, the SAM resolved, with an LVOT gradient of $10 \mathrm{~mm} \mathrm{Hg}$ and minimal MR noted. The other 6 patients who developed recurrent SAM were treated medically and 5 showed resolution before discharge, with resolution remaining at the last follow-up visit. In 1 case, significant SAM was noted at discharge. The patient was 51 years old, asymptomatic, and ambulating well. His predischarge echocardiogram revealed a hyperdynamic left ventricle with an ejection fraction estimated at $75 \%$. He was discharged home with close follow-up and returned for a repeat echocardiogram 3 weeks later. That echocardiogram showed resolution of the SAM, with no MR or LVOT obstruction. His left ventricle was also no longer hyperdynamic. 


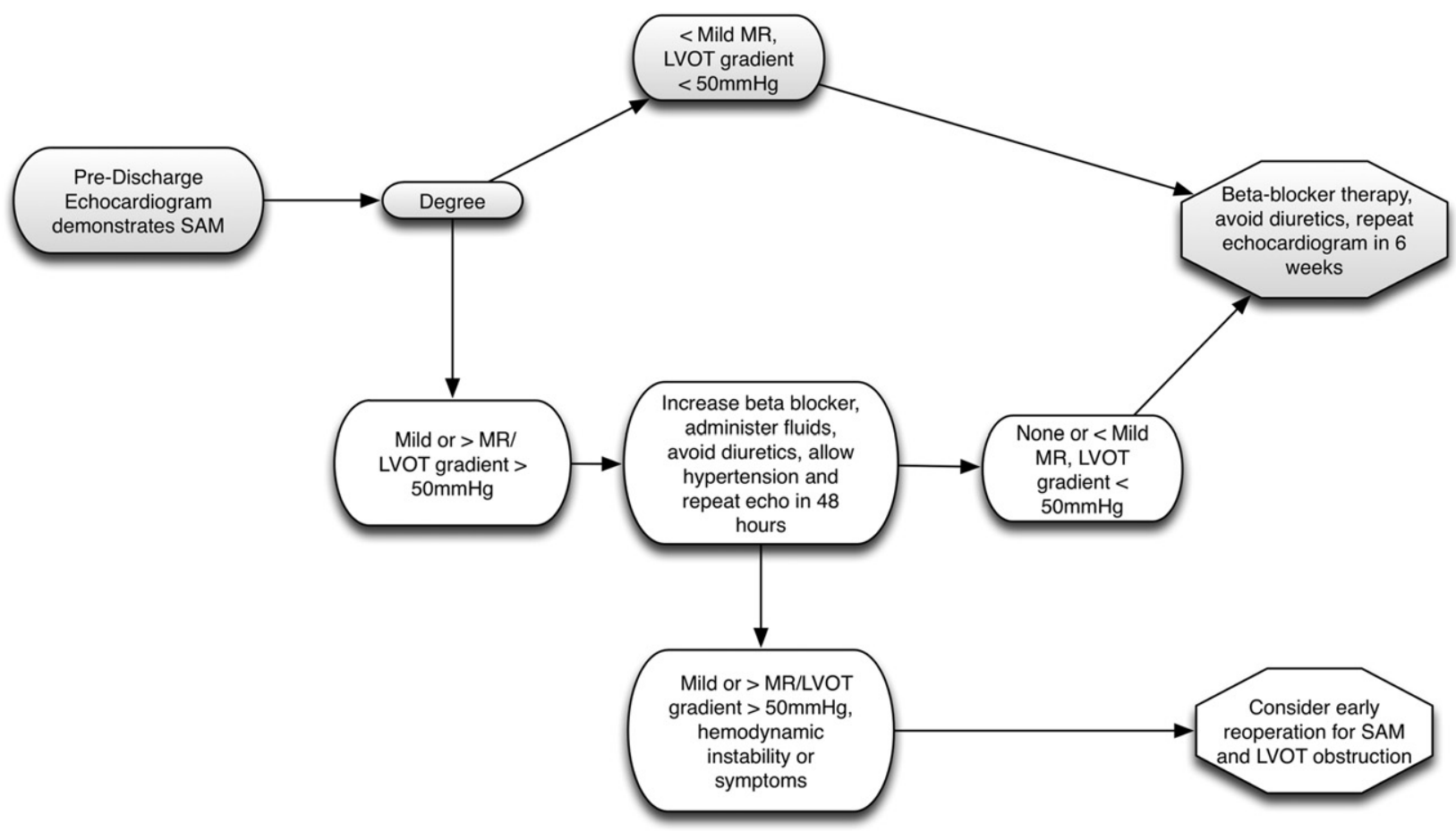

FIGURE 2. Management of postoperative systolic anterior motion.

In addition to these 7 recurrent cases of SAM, 11 new cases of SAM were identified postoperatively. These were patients in whom SAM was not reported intraoperatively by the cardiac anesthesiologist. These patients were also treated according to the SAM algorithm. In $9(81.8 \%)$ of 11 cases, SAM resolved before discharge with medical therapy. In 2 patients, significant SAM persisted on the predischarge echocardiogram despite medical therapy. Both patients were younger (age 49 and 60 years) and,

TABLE 1. Leaflet pathology and repair techniques

\begin{tabular}{lc}
\hline \multicolumn{1}{c}{ Variable } & n (\%) \\
\hline Pathology & \\
Posterior leaflet dysfunction & $45(86.5)$ \\
Anterior leaflet dysfunction & $3(5.8)$ \\
Bileaflet leaflet dysfunction & $4(7.7)$ \\
Repair technique & \\
Posterior leaflet & $44(84.6)$ \\
$\quad$ Isolated posterior resection & $15(28.8)$ \\
Posterior resection with neochordae & $7(13.5)$ \\
Posterior resection with sliding plasty & $22(42.3)$ \\
$\quad$ with or without neochordae & \\
Anterior leaflet & $4(7.7)$ \\
$\quad$ Anterior leaflet triangular resection & $2(3.9)$ \\
Chordal transfer/neochordae & $2(3.9)$ \\
Bileaflet & $4(7.7)$ \\
Posterior resection with sliding and & $4(7.7)$ \\
$\quad$ neochordae to anterior/posterior leaflet & \\
\hline
\end{tabular}

most importantly, were asymptomatic and ambulating without issue. Both patients underwent predischarge echocardiograms documenting hyperdynamic left ventricular function. On return follow-up within 6 weeks repeat echocardiogram revealed resolution of the SAM with no LVOT obstruction and their ventricles were no longer hyperdynamic.

At a mean echocardiographic follow-up of 1.3 years, no cases of significant SAM were identified. Only 1 case of mild SAM was identified. This was identified on echocardiography 3 years after surgical repair in an 83-year-old (age at follow-up) male patient who had mild SAM intraoperatively and postoperatively with a LVOT gradient less than $50 \mathrm{~mm} \mathrm{Hg}$ and no MR. At 3 years of follow-up, he was asymptomatic and the LVOT gradient was $27 \mathrm{~mm} \mathrm{Hg}$ with no MR. Of note, his septum had increased in thickness from $15 \mathrm{~mm}$ on his predischarge echocardiogram to $23 \mathrm{~mm}$ and his left ventricle was noted to be hyperdynamic with an ejection fraction estimated at $87 \%$.

During the follow-up period, there was 1 early death (noncardiac) and 2 late deaths, 1 of cardiac etiology and 1 of unknown etiology.

\section{DISCUSSION}

SAM creates a difficult management problem when observed after MVRr for degenerative disease. If it resolves intraoperatively with medical management, one is often reassured that the patient will be fine postoperatively. It is 
TABLE 2. Details of 6 patients requiring intraoperative repeat repair for systolic anterior motion

\begin{tabular}{|c|c|c|c|c|c|c|}
\hline $\begin{array}{l}\text { Pt. } \\
\text { no. }\end{array}$ & Etiology of MR & Valve lesion & Initial repair technique & Ring type and size & Repeat repair technique & $\begin{array}{c}\text { SAM } \\
\text { resolution }\end{array}$ \\
\hline 1 & Barlow's disease & $\begin{array}{l}\text { Ruptured A2 chord, } \\
\text { restricted calcified P3 } \\
\text { chord }\end{array}$ & $\begin{array}{l}\text { Triangular resection of } \\
\text { A2, annuoplasty ring }\end{array}$ & C-E Physio $40 \mathrm{~mm}$ & $\begin{array}{l}\text { Posterior leaflet } \\
\text { shortening by } \\
\text { horizontal resection at } \\
\text { annulus }\end{array}$ & Yes \\
\hline 2 & Barlow's disease & P3 chordal elongation & $\begin{array}{l}\text { P3 resection and sliding } \\
\text { plasty }\end{array}$ & C-E Physio $38 \mathrm{~mm}$ & $\begin{array}{l}\text { P1 shortening by } \\
\text { horizontal resection at } \\
\text { annulus }\end{array}$ & Yes \\
\hline 3 & Forme fruste & P2 chordal elongation & $\mathrm{P} 2$ triangular resection & C-E Physio $32 \mathrm{~mm}$ & $\begin{array}{l}\text { Triangular resection of A2 } \\
\text { tip and cutting of } \\
\text { abnormal chord; P2 } \\
\text { Gore-Tex chord to } \\
\text { lower height }\end{array}$ & Yes \\
\hline 4 & Forme fruste & P2 chordal elongation & $\begin{array}{l}\text { P2 quad resection, } \\
\text { horizontal, vertical } \\
\text { compression with } \\
\text { sliding plasty, } \mathrm{P} 2 \\
\text { neochordae }\end{array}$ & C-E Physio II 32 mm & $\begin{array}{l}\text { Shortening of P2 } \\
\text { neochordae and } \\
\text { addition of chordal } \\
\text { transfer }\end{array}$ & Yes \\
\hline 5 & Fibroelastic deficiency & P3 chordal elongation & $\begin{array}{l}\text { Chordal transfer to P3 and } \\
\text { placement of } \\
\text { neochordae to P3 }\end{array}$ & C-E Physio $30 \mathrm{~mm}$ & $\begin{array}{l}\text { Short neochordae to } \mathrm{P} 2 \\
\text { and } \mathrm{P} 3 \text { to shorten leaflet } \\
\text { height, magic suture to } \\
\text { anterior commissure }\end{array}$ & Yes \\
\hline 6 & Fibroelastic deficiency & $\mathrm{P} 2$ ruptured chord & $\mathrm{P} 2$ triangular resection & C-E Physio $26 \mathrm{~mm}$ & Short neochordae to P2 & Yes \\
\hline
\end{tabular}

Pt. no., Patient number; SAM, systolic anterior motion; C-E Physio, Carpentier-Edwards Physio Ring (Edwards Life Sciences, Irvine, Calif).

important to note that the medical management of SAM intraoperatively should not include nonphysiologic parameters such as extreme bradycardia (heart rate $<45$ beats/ $\mathrm{min}$ ) or severe hypertension (mean arterial pressure $>100$ $\mathrm{mm} \mathrm{Hg}$ ) because although they might resolve SAM in the operating room, the condition will likely return once the patient is ambulatory and no longer receiving vasoconstrictors but instead oral $\beta$-blockers. A more difficult situation arises when SAM (either mild or significant) persists after medical therapies have been instituted in the operating room. The surgeon must balance a number of factors when deciding whether to re-arrest the heart and address the SAM by rerepairing the valve. A number of factors come into play when making this decision. We have sought to provide some clarity to this decision making by presenting our algorithm for the management of SAM and our results with instituting it at our center. Our SAM incidence of $6.6 \%$ is similar to that published by other groups. ${ }^{1,2}$ This algorithm provides focused guidance on managing SAM intraoperatively and postoperatively. We must stress the importance of a technically adequate repair at the outset. Ensuring a posterior leaflet height of less than $15 \mathrm{~mm}$, ensuring a posterior displaced closure line, and avoiding an undersized annuloplasty ring are key elements to minimizing the incidence of SAM. Furthermore, being cognizant of the risk factors for SAM as previously mentioned can change one's repair strategy at the outset (eg, using a sliding plasty). Although significant SAM can resolve intraoperatively, it can-as we have shown-recur in the postoperative period. Hence, we advocate "targeted postoperative care" for patients with intraoperative SAM that improved with medical therapy, hence minimizing the incidence of recurrent SAM. We found that postoperative SAM that recurred (after resolving intraoperatively) always resolved with medical therapy and did not require surgical intervention. We advocate an immediate intervention strategy for patients who have significant SAM intraoperatively that does not resolve with intraoperative medical therapy. Some might argue that re-arresting the heart and the prolonged bypass time puts patients at increased surgical risk. However, we would argue that the repair technique required for SAM often can be done with 1 dose of cardioplegia, without removing the annuoplasty ring and just addressing the posterior leaflet height. Furthermore, the treatment of a patient in the postoperative period with myocardial dysfunction requiring inotropic support is challenging if SAM persists. It is important to note that all cases of intraoperative SAM do not resolve with medical management. Zegdi and colleagues ${ }^{10}$ reported a case of SAM after MVRr in a symptomatic patient who required repeat repair 8 years after the initial surgery. We have also had 1 case of mitral valve re-repair for SAM in a 50-year-old patient who developed New York Heart Association class II symptoms secondary to SAM with moderate MR and a LVOT gradient of $90 \mathrm{~mm} \mathrm{Hg}$ on exercise. He had undergone MVRr 5 years earlier at another 
institution. Given these findings, an aggressive, but strategic, approach to SAM should be undertaken.

In our series, we found $11(21.2 \%)$ of 52 new cases of SAM postoperatively. These were patients in whom SAM was not reported intraoperatively. We believe this can be explained by dynamic changes in left ventricular function during the postoperative period. Myocardial stunning, which was present intraoperatively, might have resolved and the increased ventricular function in the presence of an underfilled ventricle might have led to SAM. In all cases of new postoperative SAM, medical management was adequate at leading to resolution. In 2 cases of new postoperative SAM, the LVOT gradients were measured at greater than $50 \mathrm{~mm} \mathrm{Hg}$. Both of these patients had hyperdynamic left ventricles with an ejection fraction of $70 \%$ or greater postoperatively. In these 2 asymptomatic younger patients, we elected to continue medical therapy and allow their ventricles time to return to normal, which led to resolution of the SAM.

A number of surgical methods have been suggested for addressing SAM. ${ }^{11-14}$ All have the same goals of addressing the excessive posterior leaflet tissue and migrating the leaflet closure line toward the posterior annulus and hence addressing the mismatch between the mitral valve annular dimension and the amount of leaflet tissue present. Although not explicitly stated in the algorithm, mitral valve replacement for SAM is an option to consider in instances in which repeat repair has failed to alleviate SAM and the patient's comorbidities or operative duration warrant a timely resolution.

Our study had some limitations. The data were collected in a retrospective fashion, and hence some cases of SAM might have been missed. Echocardiograms were performed by a number of different technicians and read by different anesthesiologists and cardiologists, which may have led to operator biases. Our long-term data were limited to a mean of only 1.3 years; therefore, the long-term outcomes of these patients in whom SAM has resolved is not known.
Using this algorithm to address SAM in the perioperative period, we hope to provide a more systematic, guided approach to the management of SAM with proven results and solidify the value of an excellent technical repair to minimize its risk at the outset.

\section{References}

1. Brown ML, Abel MD, Click RL, et al. Systolic anterior motion after mitral valve repair: is surgical intervention necessary? J Thorac Cardiovasc Surg. 2007;133: 136-43.

2. Crescenzi G, Landoni G, Zangrillo A, et al. Management and decision-making strategy for systolic anterior motion after mitral valve repair. J Thorac Cardiovasc Surg. 2009;137:320-5.

3. Carpentier A, Adams DH, Filsoufi F. Carpentier's Reconstructive Valve Surgery. Maryland Heights: Saunders Elsevier; 2010.

4. Sherrid MV, Chaudhry FA, Swistel DG. Obstructive hypertrophic cardiomyopathy: echocardiography, pathophysiology, and the continuing evolution of surgery for obstruction. Ann Thorac Surg. 2003;75:620-32.

5. Said SM, Schaff HV, Suri RM, Greason KL, Dearani JA, Nishimura RA. Bulging subaortic septum: an important risk factor for systolic anterior motion after mitral valve repair. Ann Thorac Surg. 2011;91:1427-32.

6. Maslow AD, Regan MM, Haering JM, Johnson RG, Levine RA. Echocardiographic predictors of left ventricular outflow tract obstruction and systolic anterior motion of the mitral valve after mitral valve reconstruction for myxomatous valve disease. J Am Coll Cardiol. 1999;34:2096-104.

7. Jebara VA, Mihaileanu S, Acar C, Brizard C, Grare P, Latremouille C, et al. Left ventricular outflow tract obstruction after mitral valve repair: results of the sliding leaflet technique. Circulation. 1993;88(5 Pt 2):II30-4.

8. Anyanwu AC, Adams DH. The intraoperative "ink test": a novel assessment tool in mitral valve repair. $J$ Thorac Cardiovasc Surg. 2007;133:1635-6.

9. Adams DH, Anyanwu AC, Rahmanian PB, Abascal V, Salzberg SP, Filsoufi F. Large annuloplasty rings facilitate mitral valve repair in Barlow's disease. Ann Thorac Surg. 2006;82:2096-101.

10. Zegdi R, Carpentier A, Doguet F, Berrebi A, Khabbaz Z, Chauvaud S, et al. Systolic anterior motion after mitral valve repair: an exceptional cause of late failure. J Thorac Cardiovasc Surg. 2005;130:1453-4.

11. Quigley RL. Prevention of systolic anterior motion after repair of the severely myxomatous mitral valve with an anterior leaflet valvuloplasty. Ann Thorac Surg. 2005;80:179-82.

12. Mascagni R, Al Attar N, Lamarra M, Calvi S, Tripodi A, Mebazaa A, et al. Edge-to-edge technique to treat post-mitral valve repair systolic anterior motion and left ventricular outflow tract obstruction. Ann Thorac Surg. 2005;79: 471-4.

13. Kassem S, Jamil H. Papillary muscle-to-anterior annulus stitches: another technique to prevent systolic anterior motion after mitral valve repair. J Thorac Cardiovasc Surg. 2011;142:1278-81.

14. Sternik L, Zehr KJ. Systolic anterior motion of the mitral valve after mitral valve repair: a method of prevention. Tex Heart Inst J. 2005;32:47-9. 